\title{
The variability of organelle genomes in barley
}

Siniauskaya M.*, Ermakovich A., Liaudansky A., Davydenko O.

Institute of Genetics and Cytology, NAS, Minsk, Belarus

*email:m.sin@inbox.ru

The complete sequences of the chloroplast and mitochondrial genomes of previously unexplored wild and cultivated barley specimens were obtained by high-throughput sequencing (more than 20 samples). The database of barley organelle DNA polymorphic loci was created. Important SNP located at 3'-, 5'- UTR, promoter regions were defined according map of barley's transcriptome. A bioinformatic search for possible editing sites in the chloroplast genome was carried out. Resulting from NGS SNPs were mapped to predicted editing sites. Most probable SNPs with nonsynonymous amino acid substitutions were tested for the possibility of mRNA editing. A comparative study of cDNA and chloroplast DNA for positions 2634 (matK), 25067 (rpoCl), 49408 (ndhK), 76884 (infA), 111390 (ndhG), 29020 (rpoC2) showed that their nucleotide compositions did not differ for early seedlings stage. The variability of specific chloroplast and mitochondrial DNA regions was studied among 104 barley cultivars and lines (including 20 samples from the collection of IPK Gatersleben Genbank). 12 plasmotypes were distinguished among all studied barley's only for 7 marker regions of organelle genomes (according combained data of NGS and on individual polymorphic loci of organelle DNA). Relatively high level of organelle genomes variability was revealed. It has been shown that certain barley varieties have unique sequences of chloroplast and/or mitochondrial genes. How important this is for the functions of plants remains to be shown in the future.

This study opens up prospects for MAS, taking into account nuclearcytoplasmic interactions for the implementation of the plant genotype. Acknowledgments: The work was financed by GPNI "Biotechnology" 2019-2023 years. Subprogramme 2 "Structural and functional genomics", projects 2.29, 2.1.3. 